\title{
Evidencia serológica de infección por Hantavirus en población humana del estado de Yucatán, México.
}

\section{Artículo Original}

Ignacio Vado-Solís ${ }^{1}$, Carlos Pérez-Osorio ${ }^{1}$, Jorge Lara-Lara ${ }^{1}$, Hugo A. Ruiz-Piña ${ }^{2}$, María Cárdenas-Marrufo ${ }^{1}$, Marie Louise Milazzo ${ }^{3}$, Charles F. Fulhorst ${ }^{3}$, Jorge Zavala-Velázquez ${ }^{1}$.

${ }^{1}$ Departamento de Patología Tropical, Facultad de Medicina, ${ }^{2}$ Centro de Investigaciones Regionales “Hideyo Noguchi”, Universidad Autónoma de Yucatán. Mérida, Yucatán, México. ${ }^{3}$ Deparment of Pathology and WHO Center for Tropical Diseases, University of Texas Medical Branch, Galveston, Texas USA.

\section{RESUMEN.}

Introducción. El Síndrome Pulmonar por Hantavirus (SPH) es una zoonosis emergente en el continente Americano, caracterizado por un estado febril y rápida aparición de edema pulmonar con letalidad $>40 \%$. Es ocasionada por un virus de la familia Bunyaviridae; sus principales reservorios son roedores de la subfamilia Sigmodontinae. El propósito de este trabajo fue detectar la presencia de anticuerpos contra Hantavirus en muestras de suero humano y mamíferos silvestres y sinantrópicos en el estado de Yucatán.

Material y Métodos. Se analizaron 616 muestras de suero humano provenientes de población abierta y 92 muestras de roedores mediante la técnica de ELISA indirecta para la detección de anticuerpos contra Hantavirus.

Resultados. Se demostró la presencia de anticuerpos contra Hantavirus en cuatro muestras de humanos (0.6\%), con títulos de 1/320 a > 1/20,000; el rango de edad de las personas seropositivas fluctuó entre
2.4 a 80 años. Sin embargo, los sueros de los roedores fueron negativos.

Conclusión. La identificación de personas seropositivas a Hantavirus es la primera evidencia de que un virus cercano a los que ocasionan el SPH, está circulando en el Estado de Yucatán, México.

(Rev Biomed 2003; 14:221-225)

Palabras clave: Hantavirus, zoonosis, epidemiología, Yucatán México.

\section{SUMMARY.}

Serum evidence of Hantavirus infection in people from Yucatan Mexico.

Introduction. Hantavirus Pulmonary Syndrome (HPS) is an emerging zoonosis in the American Continent characterized by a febrile state and the sudden onset of pulmonary edema; with a fatality rate $>40 \%$. It is caused by members of the virus family

Solicitud de sobretiros: Jorge Zavala-Velázquez. Facultad de Medicina. Universidad Autónoma de Yucatán. Ave. Itzáes No.498 Col. Centro. C. P. 97000. Mérida, Yucatán, México. Tel. 52 (999) 9-24-05-54, Fax. 52 (999) 9-23-32-97; E-mail: zavala@tunku.uady.mx Recibido el 21/Julio/2003. Aceptado para publicación el 30/Octubre/2003. 
I Vado-Solís, C Pérez-Osorio, J Lara-Lara, H Ruiz-Piña, M Cárdenas-Marrufo, ML Milazzo, y col.

Bunyaviridae genus Hantavirus. Rodents are the principal hosts of the hantaviruses that cause HPS. This study was carried out to investigate the presence of hantavirus antibodies in humans and rodent reservoirs in Yucatan State, Mexico.

Material and Methods. Six hundred and sixteen human serum samples from an open population and ninety-two samples from rodents were tested by the ELISA method.

Results. Four human serum samples showed antibodies to hantavirus ( $0.6 \%$ seroprevalence). Antibody titers ranged from $1 / 320$ to $>1 / 20,000$. Age of seropositive people ranged from 2.4 to 80 years old. However none of the rodent samples showed antibodies to hantavirus.

Conclusion. The identification of people with hantavirus antibodies is the first piece of evidence that a hantavirus, antigenically related to agents of HPS, occurs in Yucatán, Mexico.

(Rev Biomed 2003; 14:221-225)

Key words: Hantavirus, zoonosis, epidemiology, Yucatan Mexico.

\section{INTRODUCCIÓN.}

Los Hantavirus constituyen un género de la familia Bunyaviridae los cuales han coevolucionado con sus reservorios naturales: roedores de las subfamilias Arvicolinae y Sigmodontinae de la familia Muridae. En ellos los virus se transmiten en forma horizontal ocasionando una infección subclínica que perdura por mucho tiempo. Los virus se eliminan a través de la saliva, orina y heces de roedores infectados; el hombre adquiere accidentalmente la enfermedad al ponerse en contacto con estas excreciones (1).

En 1993 se reportaron en el suroeste de los Estados Unidos 48 pacientes con fiebre, cefalea y mialgias seguidas al cabo de dos a tres días por tos, disnea, insuficiencia respiratoria y muerte en el 56\% de los casos; la investigación de este problema llevó a la identificación de un Hantavirus al que se le denominó Virus Sin Nombre (SNV, por sus siglas internacionales), y a la enfermedad Síndrome
Pulmonar por Hantavirus (2). A partir del reporte de esta enfermedad se han identificado en Norte, Centro y Sur América cuando menos otros 10 Hantavirus, una gran variedad de especies de roedores reservorios de éstos, y descrito síndromes muy semejantes entre sí (3-9). En México se ha demostrado la presencia de Hantavirus o anticuerpos en Peromyscus maniculatus, $P$. melanotis, $P$. hylocetes, Reithrodontomys megalotis y $R$. sumichrasti, capturados en los estados de Jalisco, D.F., Oaxaca, México, y Zacatecas $(4,10,11)$.

Se han documentado brotes esporádicos de SPH en Canadá (12), en los Estados Unidos de América, Argentina, Chile, Brasil, Paraguay, Perú, Bolivia (4), y Panamá (5); en México no se han reportado brotes de este síndrome a la fecha; sin embargo, en estudios realizados en estados del centro y norte de la República se han encontrado seroprevalencias que varían de $1.4 \%$ a $1.6 \%$ (13). El objetivo de este trabajo fue investigar la presencia de anticuerpos contra hantavirus en el Estado de Yucatán.

\section{MATERIAL Y MÉTODOS.}

Se obtuvieron del banco de sueros del Laboratorio de Patología Tropical de la Facultad de Medicina, de la Universidad Autónoma de Yucatán, 616 muestras recolectadas en el período 2000-2001 en población abierta del estado de Yucatán. Se capturaron 92 roedores con trampas tipo Sherman utilizando como cebo avena humedecida con vainilla en ranchos ganaderos, granjas porcícolas y montes cercanos a poblaciones rurales de 9 municipios del Estado durante el período febrero-agosto de 2002, los cuales fueron trasladados al laboratorio donde se procedió a la obtención de sangre y a su clasificación taxonómica.

Tanto los sueros humanos como de roedores fueron enviados simultáneamente al Departamento de Patología de la Universidad de Texas, Galveston, E.E. U.U., donde fue investigada la presencia de anticuerpos ( $\mathrm{IgG})$ reactores mediante la prueba de ELISA utilizando virus Cano Delgadito (CDGV, por sus siglas internacionales) en la forma descrita

\section{Revista Biomédica}


Hantavirus en Yucatán México.

\section{Cuadro 1}

Reactores humanos a Hantavirus en el estado de Yucatán.

\begin{tabular}{ccccc}
\hline Sexo & Edad (años) & Municipio & Ocupación & Títulos \\
\hline M & 80 & Maní & Agricultor & $>20,000$ \\
M & 37 & Hoctún & Agricultor & 320 \\
M & 2.4 & Tekit & --- & 320 \\
F & 42 & Dzoncahuich & Labores domésticas & 320 \\
\hline
\end{tabular}

previamente por Fulhorst y col.(6); el CDGV es un Hantavirus del Nuevo Mundo que cruza con otras especies de este género y que afectan a roedores de la subfamilia Sigmodontinae. El punto de corte establecido para la prueba fue de 1/320 (6).

\section{RESULTADOS.}

Solamente $4(0.6 \%)$ de las 616 muestras de humanos reaccionaron contra el antígeno utilizado; éstas se obtuvieron en municipios del centro y sur del Estado: Hoctún, Dzoncahuich, Tekit y Maní (cuadro 1). El rango de edad de esas personas fluctuó entre
2.4 a 80 años, correspondiendo a esta última el mayor título encontrado: $>20,000$.

Dos de los adultos varones seropositivos tienen en común realizar trabajos de campo; la única mujer seropositiva del grupo vive en un rancho ganadero de su localidad.

Se capturaron 92 roedores (cuadro 2) correspondientes a cinco especies de la familia Muridae, en 9 municipios del Estado. Peromyscus yucatanicus fue la especie mayormente capturada. Solamente 13 roedores (14.1\%) se capturaron en tres de los municipios donde se encontraron humanos seropositivos. Mérida y Tetiz fueron los lugares con

\section{Cuadro 2}

\section{Distribución de la captura de roedores por Municipio.}

\begin{tabular}{lccccccc}
\hline Municipio & Rr & Of & Py & Rg & Sh & Total & \% \\
\hline Mérida & 4 & 0 & 21 & 0 & 0 & 25 & 27.2 \\
Tizimín & 1 & 1 & 1 & 2 & 8 & 13 & 14.1 \\
Ucú & 0 & 0 & 19 & 0 & 0 & 18 & 19.1 \\
Tetiz & 0 & 0 & 19 & 0 & 0 & 19 & 20.6 \\
Dzoncahuich* & 1 & 0 & 5 & 0 & 0 & 6 & 6.5 \\
Maní* & 2 & 0 & 2 & 0 & 0 & 4 & 4.3 \\
Conkal & 2 & 0 & 0 & 0 & 0 & 2 & 2.1 \\
Tekit* & 2 & 0 & 1 & 0 & 0 & 3 & 3.3 \\
Muna & 0 & 0 & 1 & 0 & 0 & 1 & 1.0 \\
\hline Total & 12 & 1 & 69 & 2 & 8 & 92 & \\
\hline
\end{tabular}

$\mathrm{Rr}=$ Rattus rattus $\mathrm{Of}=$ Oligoryzomys fulvescens $\mathrm{Py}=$ Peromyscus yucatanicus. $\mathrm{Rg}=$ Reithrodontomys gracilis. $\mathrm{Sh}=$ Sigmodon hispidus.

*Municipios donde se encontraron humanos seropositivos, además de Hoctún. 
I Vado-Solís, C Pérez-Osorio, J Lara-Lara, H Ruiz-Piña, M Cárdenas-Marrufo, ML Milazzo, y col.

mayor captura ( 25 y 19 , respectivamente). Todas las muestras de los roedores fueron negativas.

\section{DISCUSIÓN.}

La detección de personas seropositivas a Hantavirus indica que estos agentes infecciosos están circulando en el Estado. La seroprevalencia encontrada $0.64 \%$, es inferior a la reportada por Flores León y col. (1.6\%) en 326 muestras de diversas regiones del país; los sueros positivos fueron recolectados en Colima, Hidalgo, Guanajuato y Distrito Federal. Los mismos autores refieren un estudio realizado en Chihuahua en 1998 donde se encontró una seroprevalencia del 1.4\% (13).

Esta virosis es más frecuente en áreas rurales, debido a la presencia de roedores silvestres, los cuales han sido señalados como reservorios naturales del virus así como componentes fundamentales de los mecanismos de transmisión al humano. Este hecho coincide con que los cuatro pacientes encontrados serológicamente positivos en el Estado de Yucatán radican en el área rural.

El haber encontrado una persona con títulos extremadamente altos, sin antecedentes clínicos, podría explicarse como un paciente en período de recuperación, o bien como una persona en la que la infección cursa con manifestaciones leves o de forma asintomática, lo cual ha sido reportado previamente $(14,15)$. La presencia de positividad en un niño de tan corta edad sugiere una transmisión intradomiciliaria $(9,16)$.

Todos los roedores capturados han sido señalados en diversas partes del mundo como reservorios naturales de Hantavirus, (excepto la especie $P$. yucatanicus); a pesar de ello, en ninguno encontramos anticuerpos que indiquen infección, lo que podría atribuirse a que la muestra fue relativamente pequeña y arbitraria y que en su gran mayoría no provino de las zonas en donde se encontraron los casos seropositivos, lo que obliga a realizar un estudio exhaustivo en esos lugares, a fin de identificar el o los reservorios implicados.

Por otra parte, el haber encontrado sujetos

\section{Revista Biomédica}

seropositivos en nuestro medio, pone de manifiesto la necesidad de realizar el diagnóstico diferencial con HPS en pacientes con neumonía atípica, ya que además de poder establecer el tratamiento oportuno, permitirá conocer el impacto real de esta zoonosis en la salud de la población del estado de Yucatán. La identificación de personas seropositivas a Hantavirus es la primera evidencia de que un virus antigénicamente cercano a aquellos que ocasionan el SPH está circulando en el sureste de México.

\section{REFERENCIAS.}

1.- Schmaaljohn C, Hjelle B. Hantavirosis: A global disease problem. Emerg Infect Dis 1997; 3:95-104.

2.- Duchin J, Koster F, Peters C, Simpson G, Tempest B, Zaki $\mathrm{S}$, et al. Hantavirus pulmonary syndrome; clinical description of disease caused by a newly recognized hemorrhagic fever virus in the southwestern United States. N Engl J Med 1994; 330:945-9.

3.- Kanerva M, Mustonen C, Vaheri A. Pathogenesis of Puumala and other hantavirus infections. Rev Med Virol 1998; 8:67-8.

4.- Mantooth S, Milazzo M, Bradley R, Hice CL, Ceballos G, Tesh R, et al. Geographical distribution of rodent-associated hantaviruses in Texas. J Vector Ecol. 2000; 26:7-14.

5.- Vincent M, Quiroz E, Gracia F, Sánchez A, Ksiazek T, Kitsutani P, et al. Hantavirus pulmonary syndrome in Panamá: Identification of novel hantaviruses and their likely reservoirs. Virology 2000; 277:14-9.

6.- Fulhorst C, Monroe M, Salas R, Duno G, Utera A, Ksiazek $\mathrm{T}$, et al. Isolation, characterization and geographic distribution of Cano Delgadito virus, a newly discovered South American hantavirus (family Bunyaviridae). Virus Res 1997; 51:159-71.

7.- Johnson A, Ksiazek T, Williams R, Bryan R, Mills J, Peters C, Popov V, et al. Laguna Negra virus associated with HPS in western Paraguay and Bolivia. Virology 1997; 238:115-27.

8.- Power A, Mercer D, Watts D, Guzmán H, Fulhorst C, Popov $\mathrm{V}$, et al. Isolation and genetic characterization of a hantavirus (Bunyaviridae: Hantavirus) from a rodent, Oligoryzomys microtis (Muridae), collected in northeastern Peru. Am J Trop Med Hyg 1999; 61:92-8. 
9.- Levis S, Morzunov S, Rowe J, Enria D, Pini N, Calderon G, et al. Genetic diversity and epidemiology of hantaviruses in Argentina. J Infect Dis 1998; 177:529-38.

10.- Suzán G, Ceballos G, Mills J, Ksiazek T, Yates T. Serologic evidence of hantavirus infection in sigmodontine rodents in Mexico. J Wildl Dis 2001; 37:391-3.

11.- Hjelle B, Anderson B, Torres-Martinez N, Song W, Gannon W, Yates T. Prevalence and geographic genetic variation of hantaviruses of New World harvest mice (Reithrodontomys): Identification of a divergent genotype from Costa Rican Reithrodontomys mexicanus. Virology 1995; 207:452-9.

12.- Verity R, Prasad E, Grimsrud K, Artsob H, Debrot M, Mieddzinski L, et al. Hantavirus pulmonary syndrome in Nothern Alberta Canada: Clinical and laboratory findings for 19 cases. Clin Infect Dis 2000; 31:942-6.

13.- Flores L, Morales J. Situación de los Hantavirus en México. Enf Infect Microbiol 2001; 21 (Supl):575.

14.- Bostik P, Winter J, Ksiazek T, Rollin P, Villinger F, Saki S, et al. Sin Nombre Virus (SNV) Ig isotype antibody response during acute and convalescent phases of hantavirus pulmonary syndrome. Emerg Infect Dis 2000; 106:184-8.

15.- Zaki S, Coffield L, Goldsmith C, Nolte K, Foucar K, Feddersen RM, et al. Hantavirus pulmonary syndrome: pathogenesis of an emerging infectious disease. Am J Pathol 1995; 146:552-79.

16,- Nuti M, Ieradi L.A., Cristaldi M, de Gibbs C.J. Jr. Prevalence of antibody to hantavirus-like virus infections in humans and Seúl-like virus infections in rodents. Arch. Virol 1990; suppl 1: 81-86. 